\title{
Influence of Process Temperatures on Blister Creation in Micro Film Insert Molding of a Dual Layer Membrane
}

\author{
Wöhner, Timo; R. Whiteside, Ben; Tosello, Guido; Hansen, Hans Nørgaard; Islam, Aminul
}

Published in:

Proceedings of the 11th International Conference on Multi-Material Micro Manufacture (4M2016) : co-organised with 10th International Workshop on Microfactories (IWMF2016)

Link to article, DOI:

10.3850/978-981-11-0749-8 715

Publication date:

2016

Document Version

Publisher's PDF, also known as Version of record

Link back to DTU Orbit

Citation (APA):

Wöhner, T., R. Whiteside, B., Tosello, G., Hansen, H. N., \& Islam, A. (2016). Influence of Process Temperatures on Blister Creation in Micro Film Insert Molding of a Dual Layer Membrane. In Proceedings of the 11th International Conference on Multi-Material Micro Manufacture (4M2016) : co-organised with 10th International Workshop on Microfactories (IWMF2016) (pp. 51-54). Research Publishing Services.

https://doi.org/10.3850/978-981-11-0749-8 715

\section{General rights}

Copyright and moral rights for the publications made accessible in the public portal are retained by the authors and/or other copyright owners and it is a condition of accessing publications that users recognise and abide by the legal requirements associated with these rights.

- Users may download and print one copy of any publication from the public portal for the purpose of private study or research.

- You may not further distribute the material or use it for any profit-making activity or commercial gain

- You may freely distribute the URL identifying the publication in the public portal 


\title{
Influence of Process Temperatures on Blister Creation in Micro Film Insert Molding of a Dual Layer Membrane
}

\author{
Timo Wöhner ${ }^{1}$, Ben R. Whiteside ${ }^{2}$, Guido Tosello ${ }^{1}$, Hans Nørgaard Hansen ${ }^{1}$, Aminul Islam ${ }^{1,3}$ \\ ${ }^{1}$ Technical University of Denmark, Department of Mechanical Engineering, Dk-2800 Kgs. Lyngby, Denmark \\ ${ }^{2}$ University of Bradford, RKT Centre Polymer MNT, BD7 1DP Bradford, United Kingdom \\ ${ }^{3}$ Technical University of Denmark, Centre for Acoustic-Mechanical Micro Systems, Dk-2800 Kgs. Lyngby, \\ Denmark
}

\begin{abstract}
In this work the suitability of a dual layer membrane, consisting of a non-woven Polypropylene (PP) support and a membrane layer made out of Polyethylene Terephthalate (PET) for Micro Film Insert Molding ( $\mu$ FIM) was investigated. The emergence of blisters at the surface of the PET-membrane layer was observed for some combinations of the process parameters used in this investigation. Since these blisters are not only an optical issue but can also deteriorate the functionality of the membrane, a blister free overmoulding of the membrane is required. In particular the mold and the barrel temperature were found to influence this blister creation. The influence of these two parameters on the amount of blisters and the blister height, characterized by the areal surface topography parameters peak material portion (Smr1) and reduced peak height (Spk), is presented in this paper.
\end{abstract}

Keywords: Film Insert Molding, Blister Creation, Process Temperatures, Micro Injection Molding

\section{Introduction}

In Film Insert Molding a preformed film is inserted in the mold and subsequently overmolded. This injection molding technique has a broad variety of applications and is mainly used to improve the surface quality of injection molded parts or for decorative purposes [1].

The use of FIM is however not limited to these standard applications. It has been reported, that FIM was successfully used to add functional features like nano-patterned structures [2] or radio frequency identification (RFID) tags [3] to injection molded parts or to overcome the mechanical issues of weldlines in glass fiber filled thermoplastic parts [4]. The work presented in this paper is part of a project which investigates another application where functionality is added by film insert molding. This project aims at injection molded methanol containers for direct methanol fuel cells where a membrane is used as film insert to enable $\mathrm{CO}_{2}$-venting from the methanol container [5].

The main focus of research in FIM lies in the change of the thermal conditions in the mold due to the differences in thermal conductivity between the mold material and the film insert. In the case of a thermoplastic film insert the thermal conductivity of the insert is much lower than that of the mold, which is usually made out of steel. This can lead to differences in the filling behavior of the mold cavity compared to standard injection molding and also results in non-uniform cooling behavior which can lead to parts with an increased warpage [6].

The warpage of FIM-parts has been the focus of a number of research works including [7], where it was found that the warpage can be reduced by an annealing step after molding when an unannealed thermoplastic film insert was used.

Further researchers in this area dealt with the morphology of semicrystalline materials used to overmold the film insert [7, 8] and the adhesion between film insert and the molded substrate [10].

In the $\mu \mathrm{FIM}$ experiments carried out in the current research work a SABEU TRAKETCH®membrane (PET23 B K320 D0,22 hl PP50) was overmolded with PP ( 579 S by SABIC). This material combination led to severe blister creation on the surface of the membrane at some combinations of the molding parameters (see Figure 1). Since the thermal effects have been found to have the most influence on the properties of FIM parts, the influence

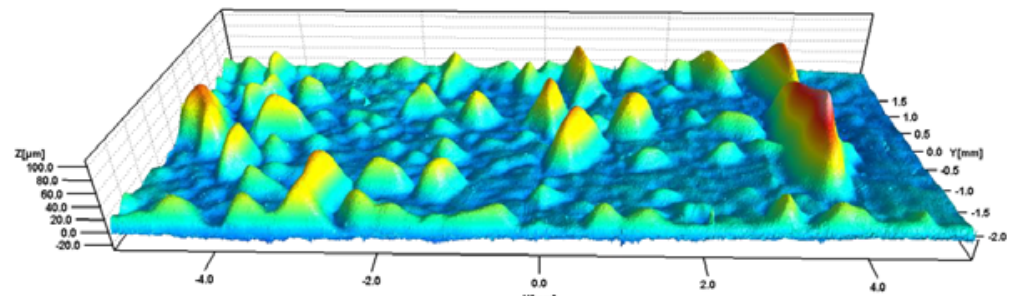

Figure 1: Blistered surface of the film insert after molding (Z-axis scale 10:1). Molding parameters: Mold temperature: $40^{\circ} \mathrm{C}$, barrel temperature: $200^{\circ} \mathrm{C}$ 

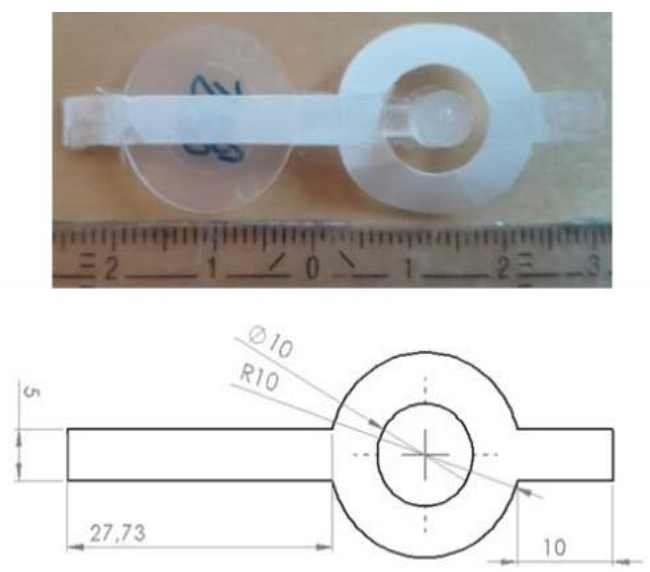

Figure 2: FIM-part including sprue, runner, gate and untrimmed film insert (top, scale unit in $\mathrm{cm}$ ). Dimensions in $\mathrm{mm}$ for film insert blank (bottom).

of mold and barrel temperature on the blister behavior is presented in this paper.

\section{Experimental Setup}

\subsection{Materials}

The film insert for these experiments was a SABEU TRAKETCH®-membrane. This membrane has a total thickness of $200 \pm 20 \mu \mathrm{m}$. It consists of a $23 \mu \mathrm{m}$ thick, porous PET-layer which has an oleophobic coating and a non-woven PP support. The pore size is $0.22 \pm 0.02 \mu \mathrm{m}$ and the pore-density is $270 \times 10^{6} / \mathrm{cm}^{2}$. PP was used as substrate material ( 579 S by SABIC). This Polypropylene grade shows a high Melt Flow Index, provides high stiffness and low warpage tendency according to the supplier. Further background information on the material selection can be found in [5].

\subsection{Part Design}

The molded part for this investigation was discshaped with a diameter of $17.25 \mathrm{~mm}$ and a thickness of $500 \mu \mathrm{m}$. In the center of the disc a $5 \mathrm{~mm}$ wide strip of the film insert was overmolded which covered the whole diameter of the disc (see Figure 2). The film insert was aligned in the mold by placing it in a recess in the movable mold half, so that the support layer was overmolded. It was fixed using adhesive tape at both ends of the film insert and additionally clamped between the mold halves.

\subsection{Molding}

For the molding experiments a WittmannBattenfeld Micro Power 15 micro injection molding machine was employed. This machine uses a screw (diameter $14 \mathrm{~mm}$ ) for plastification of the material and an injection plunger with a diameter of $5 \mathrm{~mm}$ for injecting the material.

A full factorial design of experiments (DOE) analysis was conducted to evaluate the influence of the mold and barrel temperatures. The injection speed was set to a fixed level of $100 \mathrm{~mm} / \mathrm{s}$. The

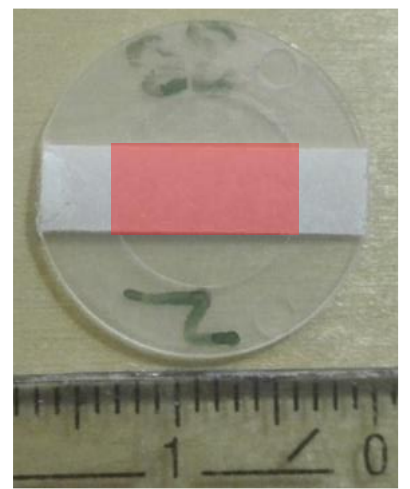

Figure 3: Measurement area of $5 \times 10 \mathrm{~mm}^{2}$ on the membrane in the center of the part (red area).

packing pressure was set to 126 bar. The packing time was set to $4 \mathrm{~s}$, which ensures an effective packing of the part since the gate freeze off is happening way before the end of the packing time.

For the mold temperature two levels have been chosen: $40^{\circ} \mathrm{C}$ and $60^{\circ} \mathrm{C}$. For the influence of the mold temperature a linear behavior is expected. This is based on the results of [6], were the influence of the mold temperature on warpage, crystallinity and crystal size was found to be linear. The temperatures were selected based on the datasheet of the material supplier, which recommends a temperature range from $15-65^{\circ} \mathrm{C}$. The lower mold temperatures of this range could not be investigated since the mold temperature was kept constant by electrical heating cartridges. Therefore the low level of the mold temperature had to be chosen above room temperature.

The levels for the barrel temperature were set to cover the range given in the processing guide for SABIC PPs. The low level was set to $200^{\circ} \mathrm{C}$, the medium level to $235^{\circ} \mathrm{C}$ and the high level to $270{ }^{\circ} \mathrm{C}$. For each possible set of parameters five parts were molded. Between each of these parts five shots without inserting a membrane were run to stabilize the process conditions. To keep the molding conditions as uniform as possible a mold opening time of 30 seconds was chosen to allow for manual insertion of the film insert.

\subsection{Metrology}

An area of $5 \times 10 \mathrm{~mm}^{2}$ on the film insert in the center of the part was measured using an Olympus Lext 4100 confocal laser scanning microscope (see Figure 3). Using this area for the measurement has the advantage that the results are not affected by boundary effects such as weldline creation which occurs close to the end of the circular cavity. For these measurements the $10 \times$ magnification lens was used in stitching mode. The obtained data was postprocessed by an image-metrology software (SPIPTM by Image Metrology $A / S$, version 6.4.3). The post processing procedure included tilt and warpage compensation and filtering of optical noise (Median $15 \times 15$ full filter). This type of non-linear filter replaces 


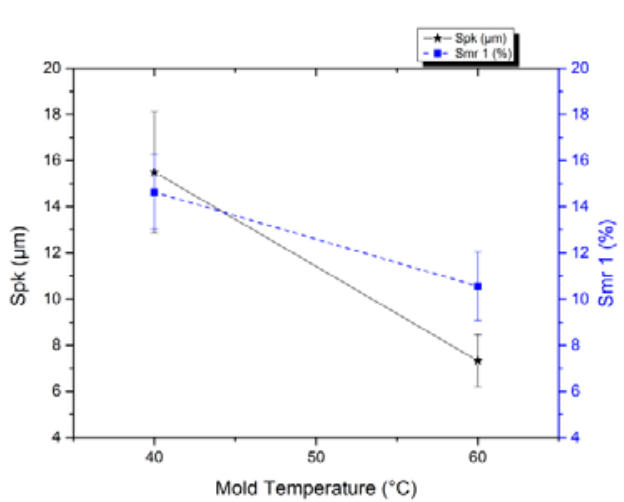

Figure 4: Influence of mold temperature on Spk and Smr1. Error bars represent the standard deviation of the mean

the height value in the center of a $15 x 15$ pixel window, by the median value of the window, if it differs too much from the median value of the window and is therefore an effective filter to reduce outliers which can be caused by e.g. speckle noise. Afterwards the area of interest was set to the boundaries of the film insert to obtain the area marked by the red box in Figure 3. Finally the zero level for the height values was set to the most frequent height and the areal surface roughness parameters were calculated. For the evaluation of the degree of blistering of the surface the reduced peak height (Spk) and the material ratio which separates the protruding peaks from the core roughness profile (Smr 1). Spk represents the average height of the peaks above the core roughness profile and can be used to evaluate the height of the blisters. The advantage of the use of this averaged parameter compared to the use of the maximum peak height $S p$ is that it compensates for outliers which can be caused by a single high blister which can be preferable to many blisters which are not as high. Smr1 represents the percentage of material above the roughness core profile and is an indicator for how much of the investigated area is related to blisters [11]

\section{Results}

In the following section the results of the DOE are presented. The points in the graphs in Figure 4 and Figure 5 indicate the mean value of all observations obtained at the indicated level of the parameter. Error bars are used to indicate the standard deviation of the mean.

The influence of the mold temperature on both Spk and Smr 1 is shown in Figure 4. An increase in mold temperature results in a decrease of both of these values. This indicates that the average blister height is being reduced with higher mold temperatures and a smaller percentage of the material is above the core roughness profile. Therefore less blistered parts can be expected at higher mold temperatures. The influence of the barrel temperature behaves similar to that of the mold temperature (see Figure 5). The plot of Spk is

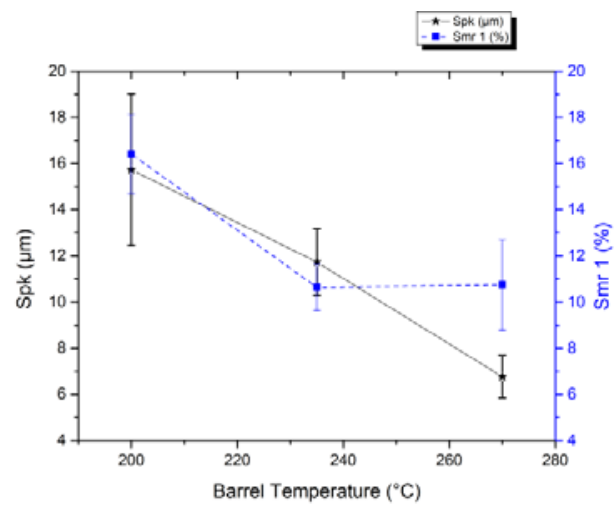

Figure 5: Influence of barrel temperature on Spk and Smr 1. Error bars represent the standard deviation of the mean

showing a linear decrease of the blister height. For the Smr 1 value a decrease is found between the low and the medium level of the barrel temperature, while the plot flattens out between the medium and the high level.

There are several possible explanations for this behavior which are mainly related to the filling and cooling behavior of the part. Increasing the barrel temperature mainly affects the viscosity of the injected substrate material and the cooling time while an increase in barrel temperature results in a slower cooling and a reduced thickness of the initial frozen layer at the interface between the substrate material and the film insert during the filling phase of the area of interest.

A reduced viscosity and frozen layer thickness could lead to an improved filling of the gaps between the PP fibers of the support material. This can lead to an improvement in the contact area for the heat exchange and therefore to a more uniform heat transfer between the substrate material and the mold. If these gaps are not filled properly the heat transfer resistance in the areas with a high density of PP fibers will be higher than in areas with a low fiber density since the contact resistance between the fibers is higher than the thermal resistance of the polymer. That is why differences in shrinkage might occur between these areas where the shrinkage will be higher in areas with a higher thermal resistance which could lead to blistering.

The reason for the non-linear behavior of Smr 1 for an increase in barrel temperature might be the crystallization of the substrate material. If the cooling rate of the substrate material is lower than the crystallization rate of the material, the shrinkage in the substrate material will be higher. This is due to the lower specific volume of the crystalline areas compared to an amorphous area of the same material. An increase of the substrate shrinkage can lead to a larger difference in the contraction between the PET of the film insert and the PP of the substrate material. This difference can lead to tensions in the film insert which can increase the tendency to create blisters.

In Figure 6 a part molded at the medium level of barrel temperature and the high level of mold 


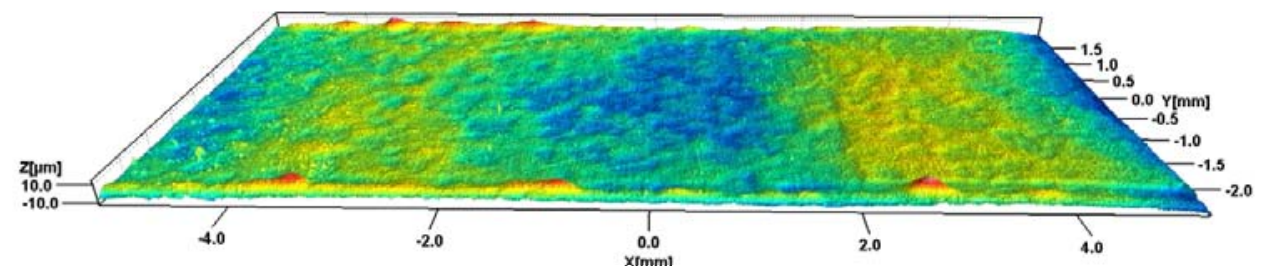

Figure 6: Part without severe blisters (Z-axis scale 10:1) Molding parameters: Mold temperature: $60^{\circ} \mathrm{C}$, barrel temperature: $235^{\circ} \mathrm{C}$

temperature is shown. The surface of the film insert is free of blisters while there are some minor blisters at the edge of the film insert. Blisters found at the edges of the film insert could have another origin than the thermal conditions. The cross-section under the film insert is reduced by the thickness of the insert compared to the rest of the cavity. Therefore the flow front velocity is lower in this area. This creates merging flow fronts at the edges of the insert leading to blister creation.

\section{Conclusions}

The influence of the process temperatures in injection molding was investigated in this paper. It was found that a high mold temperature is to be recommended to reduce the amount and height of blisters.

High barrel temperatures can reduce the blister height but have been found to have a limited effect on the Smr 1 value. It is suspected that this effect might be caused by a higher degree of crystallinity in semicrystalline materials like PP and the higher differential shrinkage between substrate material and film insert in the cooling phase.

Blisters at the edges of the film insert might be independent from the process temperatures and can be related to the hesitating flow due to the thinner cross-section in the cavity of the mold under the film insert.

It has been shown that the blister creation in micro film insert molded parts can be reduced to some minor blisters at the boundaries of the film insert, which might be solved by an improved mold design. Therefore it is expected that blister free overmolding can be achieved with the presented combination of materials by choosing the correct set of process parameters.

\section{Acknowledgements}

The funding of this project by the Danish innovation consortium "NanoEnergi" is highly acknowledged. Furthermore the support of the colleagues at the Centre for Polymer Micro and Nano Technology at the University of Bradford during the conduction of the experimental work for this paper is acknowledged.

\section{References}

[1] S. Y. Kim et al., "Residual stress and viscoelastic deformation of film insert molded automotive parts," J. Appl. Polym. Sci., 2010, vol. 118 , no. 5 , pp. 2530-2540.

[2] S. H. Kim et al., "Nanopattern insert molding.," Nanotechnology, 2010, vol. 21, no. 20, p. 205302.

[3] K. Weigelt et al., "Labeling the World: Tagging Mass Products with Printing Processes," IEEE Pervasive Comput., 2010, vol. 9, no. 2, pp. 59-63.

[4] Y. W. Leong et al., "Film insert molding as a novel weld-line inhibition and strengthening technique," Polym. Eng. Sci., 2008, vol. 48, no. 11 , pp. 2147-2158.

[5] T. Wöhner et al., "Mould Design and Material Selection for Film Insert Moulding of Direct Methanol Fuel Cell Packaging," in Proceedings of the 4MIICOMM2015 Conference, 2015, pp. 259-262.

[6] H.-L. Chen et al., "Effects of insert film on asymmetric mold temperature and associated part warpage during in-mold decoration injection molding of PP parts," Int. Commun. Heat Mass Transf., 2013, vol. 41, pp. 34-40.

[7] S. Y. Kim et al., "Molded geometry and viscoelastic behavior of film insert molded parts," J. Appl. Polym. Sci., 2009, vol. 111, no. 2, pp. 642-650.

[8] Y. W. Leong et al., "The effect of molding conditions on mechanical and morphological properties at the interface of film insert injection molded polypropylenefilm/polypropylene matrix," Polym. Eng. Sci., 2004, vol. 44, no. 12, pp. 2327-2334.

[9] J. Y. Kim et al., "Relationship between the crystallization behavior and the warpage of film-insert-molded parts," J. Appl. Polym. Sci., 2011, vol. 120, no. 3, pp. 1539-1546.

[10] Y. W. Leong et al., "Effects of the molecular orientation and crystallization on filmsubstrate interfacial adhesion in poly(ethylene terephthalate) film-insert moldings," J. Appl. Polym. Sci., 2007, vol. 104 , no. 4, pp. 2100-2107.

[11] International Organization for Standartization, "EN ISO 25178-2 Geometrical product specifications (GPS)-Surface texture: Areal Part 2: Terms, definitions and surface texture parameters." International Organization for standartization, 2012. 